\title{
Carnets
}

Revue électronique d'études françaises de l'APEF

Deuxième série - 8 | 2016

Du Français en cause aux causes du français

\section{Complexité, contextualisation, historicité : questions pour la didactique du français langue étrangère et des langues}

Jean-Louis Chiss

\section{(2) OpenEdition \\ Journals}

Édition électronique

URL : http://journals.openedition.org/carnets/1875

DOI : $10.4000 /$ carnets. 1875

ISSN : 1646-7698

Éditeur

APEF

Référence électronique

Jean-Louis Chiss, «Complexité, contextualisation, historicité : questions pour la didactique du français langue étrangère et des langues », Carnets [En ligne], Deuxième série - 8 | 2016, mis en ligne le 30 novembre 2016, consulté le 01 mai 2019. URL : http://journals.openedition.org/carnets/1875; DOI : 10.4000/carnets. 1875

Ce document a été généré automatiquement le 1 mai 2019.

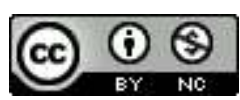

Carnets est mis à disposition selon les termes de la licence Creative Commons - Atribution - Pas d'utilisation commerciale 4.0 International. 


\title{
Complexité, contextualisation, historicité : questions pour la didactique du français langue étrangère et des langues
}

\author{
Jean-Louis Chiss
}

\section{NOTE DE L'AUTEUR}

Ce texte est récemment paru dans J-M. Defays et al, éds (2015), Transversalités. 20 ans de FLES. Faits et gestes de la didactique du français langue étrangère et seconde de 1995 à 2015

(Vol.1), Bruxelles : E.M.E., p. 79-85.

1 Les quelques éléments de réflexion qui suivent s'inscrivent dans mes intérêts actuels de connaissance (Chiss, 2010, 2013, 2014) tout en convergeant avec des débats récurrents en didactique du FLE (DFLE) et des langues (DDL) centrés sur les notions de « contexte » et de «contextualisation» qui présupposent par définition une approche complexifiée du champ et, pour moi, une pensée de l'historicité1.

2 1. Au titre de la complexité, je dirais simplement (!) que j'emploie ce terme sans référence à une théorie donnée, par exemple la "théorie des systèmes complexes" émanant des travaux en cognition et qui est à l'œuvre dans les modèles « émergentistes » de l'acquisition langagière.

Il me semble, en regardant l'empirique, que nous nous trouvons devant une extension des domaines de recherche et d'intervention de la DFLE et de la DDL. Cela me pose un problème dans la mesure où j'ai toujours plaidé pour une réduction du champ afin d'en assurer la spécificité (qui n'est pas l'équivalent de l'autonomie). Ce réductionnisme de principe ne veut pas dire, tout au contraire, l'évitement de la complexité des objets mais il ne s'agit pas non plus de prendre l'éclatement disciplinaire pour une complexification conceptuelle. La liste est longue désormais des sigles qui recouvriraient, si l'on n'y prenait 
garde, des disciplines établies : FLM, FLE, FLS, FLSco, FOS, FOU, FLI...Dans quelle mesure ces spécifications apportent-elles de l'aliment à la conceptualisation de la DFLE et de la DDL alors même que, sur le plan de la recherche, ne sont pas suffisamment travaillées les relations entre didactiques de langues différentes dans leurs dimensions intellectuelles et leurs dispositifs institutionnels? Par exemple, la mise en relation de la DFLM avec la didactique de l'anglais LM, cette même DFLM avec la didactique de l'anglais LE, la DFLE avec la didactique de l'anglais LM ou de l'anglais LE...Cette complexité ici entrevue est celle des répertoires linguistiques individuels, des référentiels de langue ou des discours grammaticaux.

4 La complexité, elle marque aussi les savoirs de toute nature à l'œuvre en DFLE et DDL : des savoirs dits "savants" aux savoirs d'expérience, y compris ceux issus des institutions éducatives, en passant par les représentations spontanées ou configurées en idéologies linguistiques complexes et contradictoires influençant les politiques linguistiques et les choix sur le marché des langues. Pour ce qui concerne les savoirs "savants", reste toujours en discussion la nature des contacts qu'ils entretiennent avec les didactiques, de la «référence » à la "contribution», le lien entre DDL et sciences du langage (SDL) demeurant pour moi décisif. Dans ce traitement des théories, on sait que le modèle de la " transposition didactique " a joué un grand rôle. Tout en reconnaissant son impact sur l'essor des didactiques, y compris celle du français et des langues, il m'est apparu contestable sur trois points décisifs : la didactisation est un processus plus complexe que la transposition didactique, malgré l'existence de ses deux étapes; la transposition didactique présuppose une compacité et une consistance des savoirs «savants » qui ne sont pas avérés dans notre domaine; elle reste un mouvement fondamentalement descendant alors même qu'il faut lui adjoindre le mouvement ascendant - des pratiques aux théories - et qu'il faut mesurer l'effet en retour du pédagogique sur le théorique, ce qui constitue, à mes yeux, l'une des tâches de toute didactique.

5 J'ajouterais à ce tableau un dernier élément de complexité: le rapport entre représentations et pratiques d'enseignement, la question des décalages que mettent en évidence les travaux sur les interactions didactiques et plus largement sur l'agir professoral, là où les démarches d'autoconfrontation et de réflexivité mettent la structuration complexe du sujet enseignant sur le devant de la scène. Un mouvement général de subjectivation, dont font partie les recherches sur les (auto)biographies langagières, affecte la DFLE et la DDL trouvant de multiples traductions dont celle de l'intérêt renouvelé pour l'affectivité.

6 2. Au titre de la contextualisation, on doit certes souligner l'omniprésence du «contexte» dans les développements actuels en DFLE et DDL mais plus encore l'émergence de la « contextualisation » dans son aspect processuel, non antinomique avec la conceptualisation mais susceptible au contraire d'y être incluse pour qu'elle ne se traduise pas en éparpillement, en fragmentation (d'un autre ordre mais tout aussi dommageable que le précédent) et ne débouche sur un relativisme généralisé et un scepticisme épistémologique. Spatialité, socialité, culturalité du contexte: la contextualisation touche toutes les dimensions théoriques, praxéologiques et axiologiques de notre discipline. J'ai essayé, avec d'autres chercheurs du DILTEC à la Sorbonne Nouvelle Paris 3 (Beacco et alii, 2005), de prendre en compte l'ensemble de ces éléments dans la tripartition cultures linguistiques/cultures éducatives/cultures didactiques.

7 Participent de ce mouvement d'ensemble de la contextualisation les enseignements littéraires et culturels/civilisationnels, les premiers parce qu'il faut compter avec la 
pluralité des littératures existantes dont celles de la francophonie et sa relation aux corpus littéraires étudiés en classe; les seconds parce qu'il s'agit de s'interroger sur l'universalisme du regard occidental, sur les stéréotypes, sur la réification de la différence en lieu et place de la pensée des formes différentes de la diversité. Dans ce trajet, le passage de la « littératie » aux « plurilittératies » est essentiel.

Mais si j'ai parlé, dans l'article de 2014, parodiant Houellebecq, d'une "extension du domaine de la contextualisation ", c'est que j'y inclus deux autres aspects : d'abord une contextualisation épistémologique, attentive à la situation des théories de la langue et des discours, aux homonymies disciplinaires (par exemple les enracinements si différents d'Applied Linguistics anglophone et de la linguistique appliquée française et francophone), à l'origine des concepts (de la " compétence de communication » aux « actes de langage »), donc aussi à la pluralité des traditions de recherche dans les différents domaines de la DDL; ensuite, une contextualisation axiologique : la DFLE et la DDL sont aux prises avec un débat et un conflit sur les valeurs portées par les savoirs et les représentations. Crise de la langue, crise dans la langue, crise dans l'enseignement de la langue et des langues.

3. Au titre de l'historicité, qui donne sens à cette complexité et à cette contextualisation, je choisirai comme illustration le plurilinguisme et le pluriculturalisme, devenus largement l'horizon commun de la DDL et des politiques linguistiques. Avec ce concept d'historicité, il ne s'agit pas seulement de recourir à l'histoire pour rendre intelligibles toutes sortes de dynamiques théoriques, méthodologiques et institutionnelles. S'il apparait nécessaire de sortir du "présentisme » et de chercher la "bonne distance ", il faut s'aviser tout à la fois d'un sentiment d'étrangeté et d'une présence du passé dans le présent, présence non univoque, ce rapport au passé se présentant sous des « régimes " multiples et parfois opposés.

Penser le plurilinguisme en France, en Europe et dans la mondialisation, c'est comprendre les limites du plurilinguisme à la française dans une histoire certes factuelle de l'homogénéisation linguistique en France, du français dans la francophonie, des français parlés et écrits mais aussi dans la relation au colonialisme et au post-colonial où la dimension comparative est requise pour ausculter les discours (francophonie vs " anglophonie » par exemple). Réalité mais aussi idéologie linguistique (ce qui n'a rien de disqualifiant), le plurilinguisme est tout entier marqué de valeurs et signe des bonheurs comme des dépossessions ou des flottements identitaires. Il est construit historiquement pour ne pas signifier le même chez les sujets et dans les sociétés d'Europe, d'Asie, d'Amérique latine ou du Maghreb. Il révèle aussi le caractère asymétrique d'une mondialisation dominée culturellement par l'Occident.

11 C'est aussi pourquoi - entre autres - la mondialisation du CECRL n'a pas, pour moi, de sens clairement assignable et que je ne comprends pas littéralement ce que veut dire l'expression «didactique du plurilinguisme » comme s'il s'agissait d'un enseignable à la même enseigne que "didactique de la grammaire» ou "didactique de l'oral». En revanche, je saisis sur le plan de l'historicité ce que peut être une éducation plurilingue et pluriculturelle, faisant écho à l'Education européenne selon le titre du roman de Romain Gary qui s'y connaissait en pluralité des langues et des cultures dans une Europe marquée par les guerres, la Shoah et le communisme. Le rapport au politique est certes déterminant mais il ne peut faire abstraction de l'histoire, pas plus de la grande que de la "petite", par exemple celle des méthodologies. Les attaques contre le CECRL sont souvent le fait d'adversaires de la construction européenne. Je m'oppose à eux, je défends 
ce cadre. Mais quand les zélateurs de ce même CECRL font passer l'approche dite « actionnelle » pour un nouveau paradigme en DDL, je m'oppose tout autant à eux.

Dans l'aire de l'enseignement et de l'apprentissage des langues, l'analyse des dispositifs institutionnels montre les historicités multiples: les départements universitaires d'« English » se sont pour une part développés dans le plurilinguisme et ont ouvert la voie aux «Cultural Studies", alors qu'en France, si l'on excepte le secteur de la littérature générale et comparée, rien de tel ne s'est produit dans les départements de Lettres. C'est pourquoi, la «crise des humanités » en France est une crise des études littéraires - et linguistiques - chez les francisants. C'est aussi pourquoi les départements de (D)FLE possèdent cette ouverture rafraichissante à laquelle les spécialistes de littérature trouvent un certain charme...

La complexité n'est pas la complication démoralisant l'effort théorique; la contextualisation n'est pas une déclinaison à l'infini des variations de toute nature finissant par invalider invariants et constantes; l'historicité n'est ni le recours à une histoire téléologique ni l'enfermement dans le présentisme. Je ne crois pas que le primat des pratiques, encore moins quand il se présente comme une mythologie du «terrain", puisse dispenser de la poursuite d'une réflexion intellectuelle attentive aux enjeux culturels de la discipline. Je ne crois pas davantage que l'inflation de nouveaux domaines (socio-didactique ou ethno-didactique) constitue une réponse aux problèmes posés en DDL et DFLE. Mutatis mutandis, William Labov répondait à la question « qu'est-ce que la sociolinguistique? » par cette formule en forme d'évidence « Mais c'est la linguistique ! ». Quant à l'« alterdidactique ", évoquée par certains, signifie-t-elle une autre didactique ou plus subtilement (?) une didactique fondée sur l'altérité ? Mais sur quelle philosophie de l'altérité ? La didactique du FLE et des langues où nous nous inscrivons est déjà occupée de ces questions. Poursuivons!

\section{BIBLIOGRAPHIE}

BEACCO, J.-C., CHISS, J.-L., CICUREL, F., VÉRONIQUE, D. (éds) (2005). Les cultures éducatives et linguistiques dans l'enseignement des langues. Paris : PUF.

CHISs J.-L. (2010). « La didactique des langues : une théorie d'ensemble et des variables ?». Le français dans le monde. Recherches et applications $\mathrm{n}^{\circ} 48, \mathrm{pp} .37-45$.

CHISs J.-L. (2013). « La didactique du français et des langues à l'épreuve de la pluralité linguistique et culturelle ». In Beacco J.-C. (dir.). Ethique et politique en didactique des langues. Autour de la notion de responsabilité. Paris : Didier, pp. 102-115.

CHISS, J.-L. (2014). » La didactique des langues comme discipline : contextualisation et historicité ", J. Aguilar, C. Brudermann, M. Leclère (éds). Langues, cultures, société : interrogations didactiques. Paris : Riveneuve Editions, pp. 299-313. 


\section{NOTES}

1. Je renvoie pour les références bibliographiques et un approfondissement des questions traitées aux articles cités supra et en particulier à Chiss (2014).

\section{RÉSUMÉS}

Ce court article, qui condense la matière de plusieurs autres, participe des débats actuels en didactique des langues sur les contextes, leur complexité et leur inscription dans l'histoire. La contextualisation entendue comme processus inclut une multiplicité de déterminations qui doivent être traitées pour elles-mêmes et globalement.

This short article, which condenses the material of several others, contributes to the current debates on language didactic concerning contexts, their complexity and their inclusion in history. Contextualization understood as a process includes multiple determinations that must be addressed apart and globally.

\section{INDEX}

Keywords : language, didactic, complexity, contextualization, historicity

Mots-clés : langue, didactique, complexité, contextualisation, historicité

\section{AUTEUR}

\section{JEAN-LOUIS CHISS}

Université Sorbonne Nouvelle Paris 3

EA 2288, DILTEC 